\title{
Infección por virus herpes humano 6 en un paciente inmunocompetente con síndrome DRESS secundario a carbamazepina
}

\author{
Sergio Álvarez, Ignacio Delama, Lucas Navajas-Galimany, Gonzalo Eymin, \\ M. Elena Ceballos y Romina Andino-Navarrete
}

Pontificia Universidad Católica de Chile. Facultad de Medicina Departamento de Dermatología (SA, LNG, RAN) Departamento de Enfermedades Infecciosas del Adulto (ID, MEC). Departamento de Medicina Interna

Conflicto de interés: Ninguno Fuente de financiamiento: Ninguna

Recibido: 2 de julio de 2015 Aceptado: 7 de abril de 2016

Correspondencia a: Romina Andino-Navarrete rominaandino@gmail.com

\section{Human herpes virus 6 infection in an inmunocompetent patient with carbamazepine-induced DRESS syndrome}

DRESS syndrome (drug reaction with eosinophilia and systemic symptoms) is an adverse life-threatening drug reaction characterized by a polymorphous rash associated with fever, lymphadenopathy and multiorgan involvement with eosinophilia. We present the case of an immunocompetent man with DRESS syndrome secondary to carbamazepine, that developed concomitantly meningoencephalitis caused by human herpes virus 6 (HHV-6), and a review of literature. The pathogenic role of HHV-6 in DRESS syndrome remains controversial. Given the diagnostic and possibly prognostic significance of HHV-6, the screening seems to be a good measure to use in the clinical management of these patients.

Key words: Drug hypersensitivity syndrome, human herpes virus 6, meningoencephalitis, anticonvulsants.

Palabras clave: Síndrome de hipersensibilidad a fármaco, virus herpes humano 6, meningoencefalitis, anticonvulsivantes.

\section{Introducción}

$\mathrm{E}$ 1 síndrome DRESS (drug reaction with eosinophilia and systemic symptoms) constituye una reacción adversa a fármacos grave con compromiso multisistémico, potencialmente mortal. Múltiples publicaciones han asociado esta entidad con una reactivación viral, siendo el virus herpes humano 6 (VHH-6) el más frecuentemente involucrado. En la actualidad, es aún controversial el rol que este virus pudiese tener tanto en el inicio como en el curso clínico de esta patología.

Presentamos el caso clínico de un hombre inmunocompetente con una meningoencefalitis por VHH-6 y un síndrome DRESS secundario a carbamazepina. Éste constituye el primer caso de esta asociación documentado en el país.

\section{Caso clínico}

Varón de 36 años, con antecedentes de una radiculopatía lumbar y un trastorno depresivo en tratamiento con carbamazepina y clomipramina iniciado seis semanas antes de su ingreso. Ingresó al hospital por un cuadro de siete días caracterizado por fiebre hasta $39^{\circ} \mathrm{C}$ asociado a calofríos, cefalea intensa, compromiso del estado general y dolor abdominal, principalmente en el hipocondrio derecho. A su ingreso estaba lúcido, febril, taquicárdico e hipertenso. Al examen físico destacaba un aumento de volumen facial, sin compromiso de la mucosa oral, asociado a un púrpura no palpable en la parte superior del tórax, con sensibilidad a la palpación en el hipocondrio derecho, sin signos de irritación peritoneal y sin adenopatías cervicales, axilares o inguinales palpables. En los exámenes de ingreso presentaba un hemograma con discreto aumento de eosinófilos ( $6 \%$ de 3.300 leucocitos) y escasos linfocitos atípicos (menos de 1\%), creatinina 1 mg/dL, LDH 172 U/L, además de una alteración de pruebas hepáticas con patrón mixto de predominio colestásico (GPT: 215 U/L, GOT: 64 U/L, GGT: 598 U/L, FA: 257 U/L, bilirrubina total: $0,72 \mathrm{mg} / \mathrm{dL}, \mathrm{INR}: 1,5)$ y aumento de la proteína $\mathrm{C}$ reactiva: $22,2 \mathrm{mg} / \mathrm{dL}$ (Valor normal $<0,5$ ). Se realizó una ecografía abdominal que evidenció una hepato-esplenomegalia asociada a una hiperecogenicidad de espacios portales. Una tomografía axial computada de abdomen y pelvis corroboró la hepato-esplenomegalia y evidenció adenopatías peri-portales. Se ingresó con el diagnóstico de una hepatitis en estudio; probablemente secundaria a medicamentos o de etiología infecciosa. Se solicitó un extenso estudio etiológico. Los hemocultivos y urocultivo resultaron negativos. Las serologías IgM para virus de hepatitis $\mathrm{A}$, antígeno de superficie para virus hepatitis $B$, anticuerpos de virus hepatitis $C$, IgM VCA de virus de Epstein Barr (VEB), citomegalovirus y VIH, fueron negativos. Se complementó estudio con RPR, IgM Toxoplasma gondii, IgM e IgG Leptospira spp., 
IgM sarampión, reacción de aglutinación para Brucella abortus/melitensis, IgG Brucella canis, IgG Bartonella hensenlae, IgG e IgM Borrelia burgorferi, IgM Mycoplasma pneumoniae fueron todas negativas. Además se realizó un panel molecular de virus respiratorios que resultó negativo. El estudio reumatológico con anticuerpos anti-nucleares, anti-citoplasmáticos, anti-músculo liso, anti-mitocondriales, anti-endomisio, factor reumatoideo, y antitransglutaminasa tisular resultó normal.

El paciente evolucionó con estabilidad clínica, sin signos de insuficiencia hepática, pero con fiebre persistente y aparición de cefalea y un exantema máculopapular morbiliforme de predominio perifolicular, en la cara y el cuello, con posterior extensión hacia el tórax, dorso, abdomen y extremidades, asociado a púrpura no palpable en extremidades inferiores. Dado que durante su evolución la cefalea se fue intensificando, se decidió realizar una punción lumbar, la cual dio salida a un líquido cefalo-raquídeo (LCR) incoloro, transparente, en cuyo análisis se encontraron 30 células $/ \mathrm{mm}^{3}, 100 \%$ mononucleares, con glucosa y proteínas normales, tinción de Gram sin bacterias y látex bacteriano negativo. El cultivo corriente también fue negativo. Se realizó una reacción de polimerasa en cadena (RPC) en LCR para enterovirus, virus herpes simplex 1-2 y virus varicela zoster que fueron negativos. Con estos hallazgos se decidió realizar estudio con RPC cualitativa para VHH-6 en sangre y LCR, que resultaron ambas positivas para VHH-6. Para completar el estudio, se tomaron biopsias de las lesiones cutáneas. El estudio histopatológico demostró una dermatitis perivascular superficial y perifolicular liquenoide con presencia de eosinófilos, compatible con una reacción adversa a medicamentos. Dada la presentación clínica, hallazgos de laboratorio y el antecedente de uso reciente de carbamazepina, fármaco frecuentemente asociado a cuadros de hipersensibilidad, se estableció el diagnóstico de síndrome DRESS en concomitancia con una meningoencefalitis aguda secundaria a una infección por VHH-6. En este caso se estableció un diagnóstico definitivo de DRESS de acuerdo a criterios RegiSCAR y DRESS atípico según criterios JSCAR. Tras la suspensión del fármaco el paciente evolucionó espontáneamente con mejoría clínica y desaparición de la cefalea. El compromiso cutáneo disminuyó con el uso de hidrocortisona tópica, sin requerir corticoesteroides sistémicos. Los exámenes de laboratorio fueron mejorando gradualmente, llegando a tener pruebas hepáticas normales al mes y medio desde el inicio del cuadro. No se encontró eosinofilia ni linfocitosis atípica $>5 \%$. Debido a su buena evolución y siendo inmunocompetente se decidió no dar tratamiento antiviral, siendo dado de alta tras nueve días de hospitalización para ser observado en forma ambulatoria. A los dos meses de su alta, el paciente ha permanecido estable, sin recurrencia de los síntomas.

\section{Discusión}

DRESS es el acrónimo en inglés de un exantema asociado a fármacos con eosinofilia y síntomas sistémicos, previamente denominado síndrome de hipersensibilidad inducido por drogas. Constituye una reacción adversa a medicamentos, potencialmente mortal, caracterizada por una erupción cutánea polimorfa asociada a fiebre, linfadenopatías y compromiso multiorgánico con eosinofilia ${ }^{1,2}$. Su incidencia se estima entre uno de cada 1.000 a uno de cada 10.000 en usuarios de fármacos anticonvulsivantes ${ }^{2,3}$. A diferencia de otras reacciones a fármacos, ésta suele presentarse hasta dos meses posterior al inicio de una terapia farmacológica, siendo más frecuente entre dos a seis semanas ${ }^{4}$. La mortalidad reportada fluctúa alrededor de $10 \%$ en adultos, siendo la falla hepática aguda la causa predominante ${ }^{2,5}$. Los anticonvulsivantes aromáticos (fenitoína, fenobarbital y carbamazepina) y las sulfonamidas son los agentes causales más frecuentes de DRESS, pero una variedad de compuestos como lamotrigina, ácido valproico, alopurinol, antiinflamatorios no esteroidales y antimicrobianos (incluyendo los antituberculosos) han sido asociados a esta entidad clínica ${ }^{6,7}$.

Desde que fue inicialmente descrito han surgido múltiples definiciones diagnósticas, las cuales han llevado a que exista más de un nombre para referirse a la misma patología. Bocquet y cols. ${ }^{5}$, introdujeron el acrónimo DRESS con el objetivo de unificar conceptos y evitar ambigüedades. Los criterios propuestos incluyeron: una erupción cutánea secundaria a un fármaco, alteraciones hematológicas, incluyendo eosinofilia mayor a $1.500 / \mathrm{mm}^{3}$ o la presencia de linfocitos atípicos y compromiso sistémico: adenopatías mayores a $2 \mathrm{~cm}$ en diámetro, hepatitis, nefritis intersticial, neumonía intersticial o carditis. En la actualidad, para definir este síndrome existen dos sistemas ampliamente utilizados. Uno de ellos fue desarrollado por el European Registry of Severe Cutaneous Adverse Reaction (RegiSCAR) ${ }^{8}$ y el segundo es del Japanese Research Committee on Severe Cutaneous Adverse Reaction (J-SCAR) ${ }^{9}$. Ambas definiciones incluyen más criterios que los propuestos inicialmente por Bocquet. Cabe destacar que uno de los criterios diagnósticos de la definición J-SCAR hace alusión al rol de la reactivación de VHH-6 en este cuadro (Tablas 1 y 2).

La patogenia del síndrome DRESS aún se desconoce. Se han propuesto varios mecanismos, destacando la predisposición genética, anomalías en el sistema enzimático responsable de la desintoxicación de medicamentos, acumulación de metabolitos reactivos y reactivaciones virales, especialmente de la familia Herpesviridae $e^{2,10,11}$. En relación a la predisposición genética para desarrollar un síndrome DRESS secundario a carbamazepina, en particular, se ha asociado a algunos alelos HLA tales 
Tabla 1. Criterios diagnósticos para DRESS según criterios de Bocquet et al. y J-SCAR ${ }^{2}$

Bocquet et al. ${ }^{1}$
Erupción cutánea por fármaco
Alteraciones hematológicas
- Eosinófilos $\geq 1,5 \times 10^{9} / L$
- Presencia de linfocitos
atípicos
Compromiso sistémico
- Adenopatía: linfonodos
$\geq 2$ cm diámetro
- Hepatitis con transaminasas
$\geq 2$ veces lo normal
- Nefritis intersticial
- Neumonitis intersticial
- Carditis

$\begin{array}{ll}\begin{array}{ll}\text { Anormalidades hepáticas (GPT } \\ >100 \mathrm{U} / \mathrm{L}) \text { o compromiso } \\ \text { de otro órgano }\end{array} & \begin{array}{l}\text { Compromiso sistémico } \\ \text { (TAC) }\end{array} \\ & \begin{array}{l}\text { - Hepato-esplenomegalia } \\ \text { (TAC) } \\ \text { - GPT 215, GOT } 64\end{array} \\ & \text { - Meningitis } \\ \text { Alteración leucocitos }(\geq 1) & \text { Alteraciones hemograma } \\ \text { - Leucocitosis }\left(>11 \times 10^{9} / \mathrm{L}\right) & \text { - Leucocitos } 3.300 \\ \text { - Linfocitos atípicos }(>5 \%) & \text { - Eosinófilos } 6 \% \\ \text { - Eosinofilia }\left(>1,5 \times 10^{9} / \mathrm{L}\right) & \text { - Linfocitos atípicos escasos } \\ \text { Linfadenopatía } & \text { RPC VHH-6 (+) } \\ \text { Reactivación de } \mathrm{VHH}-6 & \end{array}$

'Se requieren tres criterios (por lo menos uno hematológico y un compromiso sistémico). ${ }^{2}$ DRESS típico requiere los siete criterios, DRESS atípico sólo cinco.

\section{J-SCAR ${ }^{2}$ Caso actual \\ Caso actual}

Rash máculo-papular que comienza > 3 semanas de iniciado el fármaco sospechoso

Síntomas clínicos prolongados post descontinuación de fármaco causal

Fiebre $>38^{\circ} \mathrm{C}$

Comienzo seis semanas posterior al inicio de carbamazepina

Fiebre hasta $39^{\circ} \mathrm{C}$

Rash máculo-papular morbiliforme

Fiebre $>38^{\circ} \mathrm{C}$

(n)$$
\text { (1) }
$$

detectado ADN viral en el torrente sanguíneo de recién nacidos sanos seronegativos ${ }^{18}$. Constituye un patógeno ubicuo, presente en gran proporción de la población adulta en el mundo ${ }^{19}$. En niños, la infección aguda puede ser asintomática o presentarse en forma de exantema súbito. Dada su alta seroprevalencia a temprana edad (sobre 95\%), es infrecuente la primoinfección en adultos. Posterior a la infección en la niñez, el virus puede quedar latente en las células mononucleares y reactivarse durante la adultez, especialmente en pacientes inmunocomprometidos ${ }^{35}$. También se ha descrito en múltiples publicaciones la reactivación del VHH-6 en sujetos inmunocompetentes, secundario a una reacción alérgica a medicamentos y/o DRESS, lo que amplificaría el cuadro clínico y podría perpetuar los síntomas ${ }^{36}$. Es rara la presentación de meningoencefalitis por VHH-6 en una persona inmunocompetente; sin embargo, un inmunocompromiso transitorio de la respuesta inmune de células $T$, particularmente células natural killer y producción de IFN- $\alpha$, podría explicar una meningoencefalitis por VHH-6 con un sistema inmune normal $^{35}$.

Dentro de los mecanismos involucrados en su reactivación, en el contexto de síndrome DRESS, se plantea una desregulación inmune, predisposición genética o el efecto directo de ciertos fármacos ${ }^{11,20,21}$. El desencadenante pudiese ser una reacción alérgica al fármaco involucrado, lo que genera un estímulo para las células T. Dicha estimulación llevaría a la reactivación del genoma viral del VHH-6 hospedado en las células T. Una hipótesis alternativa es que el síndrome DRESS pueda desencadenarse por una reactivación silente, no clínica, del VHH-6. Ante ese escenario, las células $\mathrm{T}$ estimuladas por el virus tendrían una reacción cruzada con el medicamento, llevando a la expansión de células $\mathrm{T}$ específicas ${ }^{22,23}$.

Existen distintos métodos diagnósticos para evaluar la reactivación de VHH-6 ${ }^{18}$; sin embargo, las técnicas que permiten identificar infección activa no están estandarizadas. El aislamiento viral es altamente indicador de infección activa, pero es poco sensible y difícil de realizar. Los ensayos serológicos constan de IgM, que se elevaría a la semana de la infección aguda y por un período corto de tiempo, y de IgG, la cual eleva sus títulos significativamente en una reactivación viral ${ }^{9}$. Sin embargo, se ha detectado un alza de IgG anti-VHH-6 preexistente en infecciones por CMV y VEB, debidas posiblemente a una estimulación policlonal o una reacción antigénica cruzada, por lo que su interpretación podría ser dudosa. Por último, el estudio molecular por RPC es la técnica más sensible para detectar el genoma viral. Dado que la naturaleza ubicua de este virus y su capacidad de persistir latente y de integrar su genoma al ADN celular complican la interpretación de este examen, se promueve el uso de RPC cuantitativa como método diagnóstico más fiable. 


\begin{tabular}{|c|c|c|c|c|c|c|}
\hline Score & -1 & 0 & 1 & 2 & Min & Max \\
\hline Fiebre $\geq 38,5^{\circ} \mathrm{C}$ & No/Desc & Sí & & & -1 & 0 \\
\hline Adenopatías aumentadas de tamaño & & No/Desc & $\mathrm{Si}$ & & 0 & 1 \\
\hline $\begin{array}{l}\text { Eosinofilia } \\
\text { - Eosinófilos } \\
\text { - Eosinófilos, si leucocitos }<4.000 / \mathrm{mm}^{3}\end{array}$ & & No/Desc & $\begin{array}{c}700-1.499 / \mathrm{mm}^{3} \\
10-19,9 \%\end{array}$ & $\begin{array}{c}\geq 1.500 / \mathrm{mm}^{3} \\
\geq 20 \%\end{array}$ & 0 & 2 \\
\hline Linfocitos atípicos & & No/Desc & Sí & & 0 & 1 \\
\hline $\begin{array}{l}\text { Compromiso cutáneo } \\
\text { - Extensión de rash (> 50\% SCT) } \\
\text { - Rash sugerente de DRESS } \\
\text { - Biopsia sugerente de DRESS }\end{array}$ & $\begin{array}{l}\text { No } \\
\text { No }\end{array}$ & $\begin{array}{l}\text { No/Desc } \\
\text { Desc } \\
\text { Sí/Desc }\end{array}$ & $\begin{array}{l}\text { Sí } \\
\text { Sí }\end{array}$ & & -2 & 2 \\
\hline $\begin{array}{l}\text { Compromiso de órganos* } \\
\text { - Hígado } \\
\text { - Riñones } \\
\text { - Pulmones } \\
\text { - Muscular/corazón } \\
\text { - Páncreas } \\
\text { - Otros órganos }\end{array}$ & & $\begin{array}{l}\text { No/Desc } \\
\text { No/Desc } \\
\text { No/Desc } \\
\text { No/Desc } \\
\text { No/Desc } \\
\text { No/Desc }\end{array}$ & $\begin{array}{l}\text { Sí } \\
\text { Sí } \\
\text { Sí } \\
\text { Sí } \\
\text { Sí } \\
\text { Sí }\end{array}$ & & 0 & 2 \\
\hline Resolución $\geq 15$ días & No/Desc & Sí & & & -1 & 0 \\
\hline $\begin{array}{l}\text { Evaluación de otras causas potenciales } \\
\text { - ANA } \\
\text { - Hemocultivo } \\
\text { - Serología de VHAVHB/VHC } \\
\text { - Chlamydia/Mycoplasma pneumoniae } \\
\text { - Otra serología/RPC } \\
\text { Si ninguna positiva y } \geq 3 \text { de estas negativas }\end{array}$ & & & Sí & & 0 & 1 \\
\hline Puntaje total & & & & & -4 & 9 \\
\hline
\end{tabular}

Existen similitudes entre las manifestaciones clínicas de la reactivación por el VHH-6 y el síndrome DRESS. Ambas podrían incluir compromiso cutáneo y visceral, destacando la fiebre, hepatitis, erupción cutánea y linfadenitis histiocítica necrosante. En ocasiones, se asocia a un síndrome hemofagocítico, linfopenia y neumonitis. Todo esto ha llevado a plantear que el virus por sí mismo podría ser responsable de las manifestaciones observadas en el contexto de DRESS. De acuerdo al análisis de 62 casos reportados por Tohyama y cols. ${ }^{24}$, la aparición de síntomas como fiebre y hepatitis tiene fuerte correlato con la reactivación de VHH-6 en pacientes con DRESS. Eshki y cols. ${ }^{25}$, publicaron un caso de DRESS grave que cursó con una falla hepática fulminante y finalmente requirió de un trasplante hepático. Dicho paciente presentó un aumento significativo de los títulos de anticuerpos tipo IgG contra VHH-6 en una muestra de sangre obtenida varios meses después del trasplante. En suma, el VHH-6 podría tener un rol como co-factor del síndrome de hipersensibilidad por carbamazepina ${ }^{37}$. En vista de todo lo anterior $\mathrm{y}$, considerando que la reactivación por VHH-6 es raramente detectada en pacientes que desarrollan síndrome de hipersensibilidad a fármacos sin compromiso sistémico importante, la detección de la reactivación viral podría ser un marcador útil para el pronóstico de DRESS ${ }^{19,26,27}$.

Un factor clave en el control de la infección por VHH6 es revertir la inmunosupresión cuando sea posible; disminuyendo las dosis de fármacos inmunosupresores o iniciando la terapia anti-retroviral en pacientes con infección por VIH. Sin embargo, esta opción no siempre es posible o atingente al caso, por lo que en ciertas circunstancias se hace necesario el uso de antivirales. Los antivirales que han demostrado tener actividad in vivo e in vitro contra VHH-6 son ganciclovir, cidofovir y foscarnet. El mecanismo de acción sería similar al descrito para $\mathrm{CMV}^{38}$. Las indicaciones de tratamiento se basan principalmente en la demostración de infección activa del virus con cargas virales elevadas, asociada a un cuadro clínico grave concomitante y excluyendo otras etiologías, principalmente $\mathrm{CMV}^{39}$. Sin embargo, no existen estudios clínicos randomizados que validen las indicaciones de 
tratamiento por infección aguda por VHH-6, aunque ésta es recomendada en casos de encefalitis en niños receptores de trasplante de precursores hematopoyéticos ${ }^{38}$. En gran parte de los reportes de casos de encefalitis por VHH- 6 en individuos inmunocompetentes no se les administró tratamiento específico; en otros se describe el uso de aciclovir, cidofovir o ganciclovir. La mayoría de los casos presentó recuperación completa o parcial del compromiso neurológico, un paciente presentó crisis convulsivas persistentes y dos pacientes fallecieron ${ }^{40}$.

En la actualidad, el rol patogénico del VHH-6 en la aparición de síntomas clínicos y exacerbaciones de pacientes con DRESS sigue siendo un tema de debate y su búsqueda activa en pacientes que cursan con este síndrome es controversial ${ }^{4}$. En nuestro paciente, los síntomas neurológicos pueden ser atribuidos a la infección activa por VHH-6 en LCR, como también corresponder a una manifestación más de la reacción de hipersensibilidad propia del síndrome DRESS. Esto último es planteable dada la mejoría clínica precoz tras la suspensión del fármaco sospechoso, sin mediar tratamiento antiviral. Si bien dicha presentación es menos frecuente, la meningitis y la meningoencefalitis aséptica asociado a fármacos se han relacionado con el uso de anticonvulsivantes como carbamazepina y lamotrigina ${ }^{41,42}$.

Nuestro laboratorio no cuenta actualmente con serología ni con RPC cuantitativa para VHH-6; sin embargo, planteamos que el aislamiento del genoma viral tanto en la sangre, como en el LCR de nuestro paciente, asociado al cuadro clínico y el diagnóstico de DRESS hacen posible suponer que este virus sí habría tenido un rol activo en su enfermedad.

\section{Conclusión}

Este artículo constituye el primer caso documentado en Chile de un paciente con síndrome DRESS y meningoencefalitis concomitante por VHH-6. En nuestro país existen reportes aislados y algunas series de casos de pacientes con síndrome DRESS, destacando la asociación principalmente a fármacos anticonvulsivantes ${ }^{43-45}$. Una publicación reciente reportó un caso de meningoencefalitis aséptica en una paciente que desarrolló un síndrome DRESS por lamotrigina ${ }^{45}$. Cabe destacar que en dicho caso no se realizó estudio para identificación de VHH-6 por lo que no es posible descartar un posible rol etiológico en el cuadro.

El caso presentado y los datos publicados soportan el rol del VHH-6 como co-factor para la sensibilización y subsecuente manifestación de un síndrome de hipersensibilidad a fármacos. La reactivación del VHH-6 en la patogénesis del síndrome de hipersensibilidad, podría prolongar la duración de la enfermedad y causar cuadros clínicos graves como encefalitis ${ }^{36,37}$.

Dado los últimos reportes sobre la importancia diagnóstica, pronóstica, y potenciales beneficios de la terapia antiviral en DRESS refractarios a tratamientos convencionales, la búsqueda dirigida del virus se plantea como una alternativa a evaluar dentro del estudio de estos pacientes.

\section{Resumen}

El síndrome DRESS (drug reaction with eosinophilia and systemic symptoms) constituye una reacción adversa a fármacos, potencialmente mortal, caracterizada por una erupción cutánea polimorfa asociada a fiebre, linfadenopatías y compromiso multiorgánico con eosinofilia. Presentamos el caso clínico de un hombre inmunocompetente con un síndrome DRESS secundario a carbamazepina que cursó concomitantemente con una meningoencefalitis por virus herpes humano 6 (VHH-6). El rol patogénico del VHH-6 en el síndrome DRESS sigue siendo controversial; sin embargo, dada la importancia diagnóstica y eventualmente pronóstica de la infección por $\mathrm{VHH}-6$, su tamizaje sería recomendable dentro del estudio de estos pacientes.

\section{Referencias bibliográficas}

1.- Sullivan J R, Shear N H. The drug hypersensitivity syndrome. Arch Dermatol 2001; 137: 357-64.

2.- Husain Z, Reddy B Y, Schwartz R A. DRESS syndrome: Part I. Clinical perspectives. J Am Acad Dermatol 2013; 68: 693.e1-14.

3.- Gennis M A, Vemuri R, Burns E A, Hill J V, Miller M A, Spielberg S P. Familial occurrence of hypersensitivity to phenytoin. Am J Med 1991; 91: 631-4.

4.- Kano Y, Ishida T, Hirahara K, Shiohara T. Visceral involvements and long-term sequelae in drug-induced hypersensitivity syndrome.
Med Clin North Am 2010; 94: 743-59.

5.- Bocquet H, Bagot M, Roujeau J C.

Drug-induced pseudolymphoma and drug hypersensitivity syndrome (Drug Rash with Eosinophilia and Systemic Symptoms: DRESS). Semin Cutan Med Surg 1996; 15 : 250-7.

6.- Wolf R, Matz H, Marcos B, Orion E. Drug rash with eosinophilia and systemic symptoms vs toxic epidermal necrolysis: the dilemma of classification. Clin Dermatol 2005; 23: 311-4.

7.- Wolf R, Orion E, Marcos B, Matz H. Life-threatening acute adverse cutaneous drug reactions. Clin Dermatol 2005; 23:
171-81.

8.- Kardaun S H, Sidoroff A, Valeyrie-Allanore L, Halevy S, Davidovici B B, Mockenhaupt M, et al. Variability in the clinical pattern of cutaneous side-effects of drugs with systemic symptoms: does a DRESS syndrome really exist? Br J Dermatol 2007; 156: 609-11.

9.- Shiohara T, Lijima M, Ikezawa Z, Hashimoto K. The diagnosis of a DRESS syndrome has been sufficiently established on the basis of typical clinical features and viral reactivations. Br J Dermatol 2007; 156: 1083-4.

10.- Roujeau J C. Clinical heterogeneity of drug hypersensitivity. Toxicol 2005; 209: 123-9.

11.- Descamps V, Valance A, Edlinger C, 
Fillet A M, Grossin M, Lebrun-Vignes B, et al. Association of human herpesvirus 6 infection with drug reaction with eosinophilia and systemic symptoms. Arch Dermatol 2001; 137: 301-4.

12.- Kashiwagi M, Aihara M, Takahashi Y, Yamazaki E, Yamane Y, Song Y, et al. Human leukocyte antigen genotypes in carbamazepine-induced severe cutaneous adverse drug response in Japanese patients. J Dermatol 2008; 35: 683-5.

13.- Pirmohamed M, Lin K, Chadwick D, Park B K. TNF alpha promoter region gene polymorphisms in carbamazepine hypersensitive patients. Neurology 2001; 56: 890-6.

14.- Anjum N, Polak M E, Ardern-Jones M, Cooper H L. Presence of the HLA-A*3101 allele in a familial case of drug reaction with eosinophilia and systemic symptoms, secondary to carbamazepine. Clin Exp Dermatol 2014; 39: 307-9.

15.- Chiou C C, Yang L C, Hung S I, Chang Y C, Kuo T T, Ho H C, et al. Clinicopathological features and prognosis of drug rash with eosinophilia and systemic symptoms: a study of 30 cases in Taiwan. J Eur Acad Dermatol Venereol 2008; 22: 1044-9.

16.- Fernando S L, Henderson C J, O’Connor K S. Drug-induced hypersensitivity syndrome with superficial granulomatous dermatitis-a novel finding. Am J Dermatopathol 2009; 31: 611-3.

17.- Straus S E. Human Herpesvirus types 6 and 7. En Mandell GL, Bennett JE, Dolin R. Mandell, Douglas and Bennett's Principles and Practice of Infectious Diseases, 6th ed. Philadelphia: Churchill Livingstone Elsevier; 2005, p. 1821-5.

18.- Avendaño L. Virus herpes. En Avendaño L, Ferrés M, Spencer E. Virología Clínica. Santiago. Ed. Mediterráneo. 2011. P. 213-40.

19.- Hashimoto K, Yasukawa M, Tohyama M. Human herpesvirus 6 and drug allergy. Curr Opin Allergy Clin Immunol 2003; 3: 255-60.

20.- Cacoub P, Musette P, Descamps V, Meyer O, Speirs C, Finzi L, et al. The DRESS syndrome: a literature review. Am J Med 2011; 124: 588-97.

21.- Mardivirin L, Valeyrie-Allanore L, Branlant-Redon E, Beneton N, Jidar K, Barbaud A, et al. Amoxicillin-induced flare in patients with DRESS (drug reaction with eosinophilia and systemic symptoms): report of seven cases and demonstration of a direct effect of amoxicillin on human herpesvirus 6 replication in vitro. Eur J Dermatol 2010; 20 : 68-73.

22.- Kano Y, Hiraharas K, Sakuma K, Shiohara T. Several herpesviruses can reactivate in a severe drug-induced multiorgan reaction in the same sequential order as in graft-versus-host disease.
Br J Dermatol 2006; 155: 301-6.

23.- Shiohara $T$, Inaoka M, Kano Y. Drug-induced hypersensitivity syndrome (DIHS): a reaction induced by a complex interplay among herpesviruses and antiviral and antidrug immune responses. Allergol Int 2006; 55: 1-8.

24.- Tohyama M, Hashimoto K, Yasukawa M, Kimura H, Horikawa T, Nakajima $\mathrm{K}$, et al. Association of human herpesvirus 6 reactivation with the flaring and severity of drug-induced hypersensitivity syndrome. Br J Dermatol 2007; 157: 934-40.

25.- Eshki M, Allanore L, Musette P, Milpied B, Grange A, Guillaume J C, et al. Twelve-year analysis of severe cases of drug reaction with eosinophilia and systemic symptoms: a cause of unpredictable multiorgan failure. Arch Dermatol 2009; 145: 67-72.

26.- Shiohara T, Kano Y. A complex interaction between drug allergy and viral infection. Clin Rev Allergy Immunol 2007; 33: 124-33.

27.- Suzuki Y, Inagi R, Aono T, Yamanishi K, Shiohara T. Human herpesvirus 6 infection as a risk factor for the development of severe druginduced hypersensitivity syndrome. Arch Dermatol 1998; 134: 1108-12.

28.- Gentile I, Talamo M, Borgia G. Is the druginduced hypersensitivity syndrome (DIHS) due to human herpesvirus 6 infection or to allergy-mediated viral reactivation? Report of a case and literature review. BMC Infect Dis 2010; 10: 49 .

29.- Aihara Y, Ito SI, Kobayashi Y, Yamakawa Y, Aihara M, Yokota S. Carbamazepine-induced hypersensitivity syndrome associated with transient hypogammaglobulinaemia and reactivation of human herpesvirus 6 infection demonstrated by real-time quantitative polymerase chain reaction. Br J Dermatol 2003; 149: $165-9$

30.- Descamps V, Mahe E, Houhou N Abramowitz L, Rozenberg F, Rnager- Rogez S, et al. Drug-induced hypersensitivity syndrome associated with Epstein-Barr virus infection. Br J Dermatol 2003; 148: 1032-4.

31.- Aihara M, Sugita Y, Takahashi S, Nagatani T, Arata S, Takeuchi K, et al. Anticonvulsant hypersensitivity syndrome associated with reactivation of cytomegalovirus. Br J Dermatol 2001; 144: 1231-4.

32.- Kano Y, Inaoka M, Shiohara T. Association between anticonvulsant hypersensitivity syndrome and human herpesvirus 6 reactivation and hypogammaglobulinemia. Arch Dermatol 2004; 140: 183-8.

33.- Seishima M, Yamanaka S, Fijisawa T, Tohyama M, Hashimoto K. Reactivation of human herpesvirus (HHV) family members other than HHV-6 in drug-induced hypersensitivity syndrome. Br J Dermatol 2006;
155: 344-9.

34.- Criado P R, Criado R F, Avancini J M, Santi C G. Drug reaction with eosinophilia and systemic symptoms (DRESS)/drug-induced hypersensitivity syndrome (DIHS): a review of current concepts. An Bras Dermatol 2012; 87: 435-49.

35.- Torre D, Mancuso R, Ferrante P. Pathogenic mechanisms of meningitis/encephalitis caused by human herpesvirus- 6 in immunocompetent adult patients. Clin Infect Dis 2005; 41: 422-3.

36.- Matsuda K, Ohnuma T, Fukuta M, Kawai M, Suzuki T, Ogawa H, et al. Case reports and literature review: the association between reactivation of human herpes virus- 6 and peripheral white blood cell count in patients with carbamazepine-induced hypersensitivity syndrome. Prog Neuropsychopharmacol Biol Psychiatry 2006; 30: 751-4.

37.- Zeller A, Schaub N, Steffen I, Battegay E, Hirsch H, Bircher AJ. Drug hypersensitivity syndrome to carbamazepine and human herpes virus 6 infection: case report and literature review. Infection 2003; 31: 254-6.

38.- Agut H, Bonnafous P, Gautheret-Dejean A, Laboratory and clinical aspects of human herpesvirus 6 infections. Clin Microbiol Rev 2015; 28: 313-35.

39.- Agut H. Deciphering the clinical impact of acute human herpesvirus 6 (HHV-6) infections. J Clin Virol 2011; 52: 164-71.

40.- Birnbaum T, Padovan C, Sporer B, Rupprecht T, Ausserer H, Jaeger G, et al. Severe meningoencephalitis caused by human herpesvirus 6 type $\mathrm{B}$ in an immunocompetent woman treated with ganciclovir. Clin Infect Dis 2005; 40: 887-9.

41.- Maniyar F, Rooney C, Lily O, Bazaz R. Anticonvulsant hypersensitivity syndrome presenting as aseptic meningitis. J Neurol. 2009; 256: $1190-1$.

42.- Simms K M, Kortepeter C, Avigan M. Lamotrigine and aseptic meningitis. Neurology 2012; 78: 921-7.

43.- David P, Lackington I, Naranjo V, Oporto S, Leal C, Mena A. Actualización y presentación de serie clínica de síndrome de hipersensibilidad a fármacos anticonvulsivantes aromáticos 0 DRESS. Rev Chilena Epilepsia 2013; 13: 25-37.

44.- Rojas C, Méndez L, Arab J P, Barrera F, Soza A, Nervi F, et al. Síndrome de Dress. Eritema cutáneo, fiebre y hepatitis asociado a lamotrigina. Reporte de dos casos y revisión de la literatura. Gastroenterol Latinoam 2010; 21 : 357-62.

45.- Reyes P, Silva P. Meningoencefalitis aséptica como manifestación de un síndrome de rash con eosinofilia y síntomas sistémicos asociados a drogas por lamotrigina. Rev Chil Neuro-Psiquiat 2014; 52: 37-41. 